\title{
Velocity Calibration of Doppler Current Profiler Transducers
}

\author{
Marc Le Menn ${ }^{1, *(\mathbb{D} \text { and Steffen Morvan }}{ }^{2}$ \\ 1 Service Hydrographique et Océanographique de la Marine (Shom), CS 92803, 29228 Brest CEDEX 2, France \\ 2 Ecole Nationale Supérieure de d'Électrotechnique, d'Électronique, d'Informatique, d'Hydraulique et des \\ Télécommunications (ENSEEIHT), 31000 Toulouse, France; Steffen.morvan@gmail.com \\ * Correspondence: marc.lemenn@shom.fr
}

Received: 7 October 2020; Accepted: 26 October 2020; Published: 28 October 2020

\begin{abstract}
Doppler current profilers are used in oceanography to measure oceanic circulation but also in hydrology to calculate the flow of rivers. They allow the retrieval of water mass profiles in terms of velocity and direction. Direction is obtained via an electronic compass and tilt sensors, while velocity is obtained by measuring Doppler pulse shifts back-scattered by particles located in water cells allocated along the instrument's measurement range. Current meters are usually tested in towing basins or hydrodynamic channels, but these facilities present limits in terms of the measurement range, particles concentration and time costs. This paper presents a novel method developed to test the trueness of these velocity measurements in the laboratory, along with the uncertainty of this test and the results obtained with current meters and stand-alone profilers. The method is based on the measurement of the frequency of pulses emitted by each transducer of the instrument independently, and on the simulation of received echoes by a variable frequency sinusoidal signal.
\end{abstract}

Keywords: Doppler effect; current meter; profiler; calibration; velocity measurements

\section{Introduction}

Oceans control a big part of the Earth's climate through ocean-atmosphere exchanges, phenomena such as El-Niño or great cycles and oceanic currents. Measuring current is essential to build current charts useful for navigation, 3D models of oceanic circulation or more recently, to improve the efficiency of submarine tidal turbines.

Some ten years ago, rotor current meters were replaced by Doppler effect acoustic current meters. As the marine environment is favorable to acoustic wave propagation, the arrival time of pulses reflected by particles led to the creation of current profilers. Water-column velocity profiles can be obtained placing profilers under the hull of oceanographic boats and directed towards the seabed, or placed in cages deposited on the seabed and directed towards the surface, on mooring cables or towed from a boat. Their range, which depends on their wavelength, extends from a few meters to several hundreds of meters, according to the particles concentrations.

Depending on the pulses return times, these profiles are artificially divided into measurement cells by the instrument's software, which gives average velocity values per cell, in relation with the measured Doppler shifts.

For shipmounted instruments, several publications have been written to correct bias of alignment, sensitivity [1,2], scaling factor [3], and ship velocity measurement [4,5]. For instruments fitted on mooring cables, a method has been proposed to quantify the influence of mooring line motion on current measurements [6] and for towed profilers, a publication shows a method to correct compass bias induced by ship vicinity [7]. In the field of river hydrology, quality assurance tests [8], laboratory intercomparisons [9] or validation by bottom track in towing basins $[10,11]$ have been 
proposed. In oceanography, Doppler profiler ranges generally extend from approximately ten to several hundred meters, making controls in towing basins impossible. Moreover, the number of standalone instruments used in hydrographic and oceanographic centers makes this technique difficult to implement. Thus, over the past years, calibrating or simply testing these long range instruments has been an untreated problem.

At Shom, a platform was built and brought into service in 2012, to allow the calibration, within their instrument usage configuration, of the electronic compasses and tilt sensors installed in current meters and current profilers [12-14]. These compasses are used to retrieve the directions of profilers relative to the magnetic north, their three transducers being used to retrieve the direction of currents in the instrument referential. Compasses can be perturbed by the other instruments or battery packs mounted in cages, leading to measurement errors that need to be determined and corrected. Figure 1 shows a signature profiler mounted in a mooring cage, on the calibration platform. It remained to find a method to determine the trueness of velocity measurements.

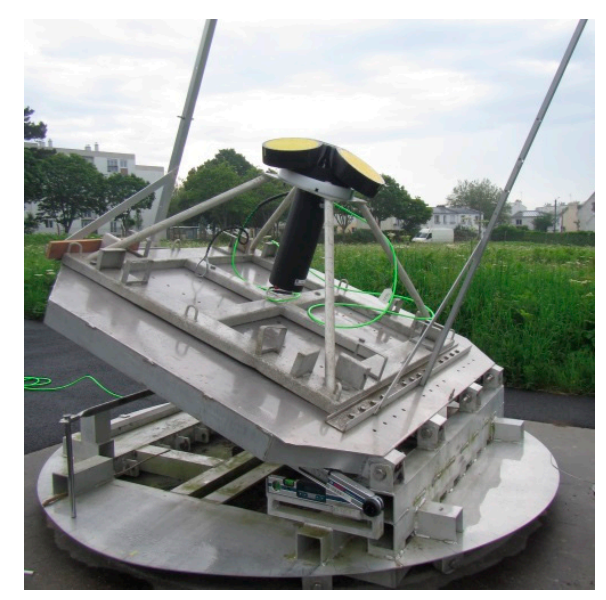

Figure 1. Photo of a Nortek Group Signature profiler (in black) on the compass calibration platform. The profiler is mounted in his mooring cage. The assembly is aligned on one of the axes of the platform, which is turned manually for heading calibration. It can be tilted for tilt sensors calibration. A reference numerical protractor is used to measure the tilt angle.

For rotor current meters, this calibration was performed in open tanks $[15,16]$ or hydrodynamic channels [17], though generally to maximum velocities included in the range 1-3 m/s. For Doppler current meters, the low particle concentration in these facilities is a problem, and by taking into account the profiling range of profilers used in oceanography, this method cannot be applied. It was still possible to carry out intercomparisons at sea as carried out in rivers by Boldt and Oberg in 2015 [18], but these intercomparisons are expensive, difficult to organize, and allow only one part of the velocity range of instruments to be tested. For rivers again, Hening Huang proposed in 2018 [19] a theoretical and a semiempirical model calibrated on transect datasets, to estimate the uncertainty of streamflow measurements made by an acoustic Doppler current profiler (ADCP) mounted on a moving platform. This method could be applied to oceanography using vessel-mounted ADCPs, though it is not adapted to standalone Teledyne RD Instruments ADCPs or equivalent instruments of Nortek group called AQPs for 'AQuadopp Profilers'.

The idea came to perfect a test using an acoustic transducer attached to the profiler transducers and linked to a frequency generator. This allows echoes received by the profiler to be simulated. The exploitation of the Doppler effect formula and of the speeds sensed by the instrument has allowed a test method of the device's measurement channels to be perfected. A calibration bed remained to be developed. 'Calibration' should be taken here as the application of the first step of the definition given in reference [20], which is: "operation that, under specified conditions, in a first step, establishes a relation between the quantity values with measurement uncertainties provided by measurement 
standards and corresponding indications with associated measurement uncertainties and, in a second step, uses this information to establish a relation for obtaining a measurement result from an indication". The test bed allows the establishment of a relation between measurement standard values whose measurement uncertainties are assessed, and values of the transducer under test with associated measurement uncertainties, to obtain a measurement result. At this time, instead of rigorously applying the second step of the definition, the calculated uncertainty allows the measured shifts to be qualified as negligible or not. When they are not negligible, this generally means that the instrument transducer has suffered a malfunction and must be repaired, preventing the use of the relationship between the reference values and the values of the transducer under test to correct the measured values.

This method opens the way for quick testing and metrological validation of current profiler transducers before their use at sea, which was previously impossible. This allows each transducer's response to be retrieved and possibly corrected independently, though it does not take into account any bias related to transducer slope imperfections that could occur due to manufacturing defects. We consider this error to be constant and corrected by the manufacturer with a custom transformation matrix if necessary (see Section 2 for further explanations).

\section{Operating Principles of Doppler Current Profilers}

Current profilers compute velocities $\left(V_{1}, V_{2}\right.$, and $\left.V_{3}\right)$ obtained by Doppler shift measurements in their beam axes (radial velocities). The transducers are tilted $20^{\circ}, 25^{\circ}$, or $30^{\circ}$ (angle $\beta$ ). It is thus possible to calculate velocities $\left(V_{x}, V_{y}\right.$, and $\left.V_{z}\right)$ in their own referential [21]:

$$
\left[\begin{array}{l}
V_{x} \\
V_{y} \\
V_{z}
\end{array}\right]=\left[\begin{array}{ccc}
\frac{2}{3 \sin (\beta)} & \frac{-1}{3 \sin (\beta)} & \frac{-1}{3 \sin (\beta)} \\
0 & \frac{-1}{\sqrt{2} \sin (\beta)} & \frac{1}{\sqrt{2} \sin (\beta)} \\
\frac{1}{3 \cos (\beta)} & \frac{1}{3 \cos (\beta)} & \frac{1}{3 \cos (\beta)}
\end{array}\right]\left[\begin{array}{l}
V_{1} \\
V_{2} \\
V_{3}
\end{array}\right]
$$

They are equipped with 'flux-gate', 'Hall effect', or magneto-resistive compasses to retrieve the amplitude of current components $(U, V$, and $W)$ in reference to magnetic north (angle $\Omega$ ), and considering the magnetic declination, in relation to true north (2). Moreover, their inclination is corrected due to a tilt sensor measuring roll and pitch angles $\psi$ and $\theta$. According to reference [22], between 1 and 12 equations can be found to describe a rotation in the three dimensions. The equation used for the profilers corresponds to Equation (2), in which $C=\cos$ and $S=\sin$ [23]:

$$
\left[\begin{array}{c}
U \\
V \\
W
\end{array}\right]=\left[\begin{array}{ccc}
C_{\psi} C_{\Omega} & \left(-S_{\psi} S_{\theta} C_{\Omega}+C_{\theta} S_{\Omega}\right) & \left(S_{\psi} C_{\theta} C_{\Omega}+S_{\theta} S_{\Omega}\right) \\
-C_{\psi} C_{\Omega} & \left(S_{\psi} S_{\theta} S_{\Omega}+C_{\theta} S_{\Omega}\right) & \left(-S_{\psi} C_{\theta} S_{\Omega}+S_{\theta} C_{\Omega}\right) \\
-S_{\psi} & -C_{\psi} S_{\theta} & C_{\psi} C_{\theta}
\end{array}\right]\left[\begin{array}{c}
V_{x} \\
V_{y} \\
V_{z}
\end{array}\right]
$$

Speeds $\left(V_{1}, V_{2}\right.$, and $\left.V_{3}\right)$ are obtained after the detection of echoes resulting from the reflection of pulses on the successive layers of particles. To improve measurement trueness, pulses are repeated at a frequency $f_{r}$. Figure 2 shows the pulse trains transmitted by a Nortek Group $2 \mathrm{MHz}$ AQP. 


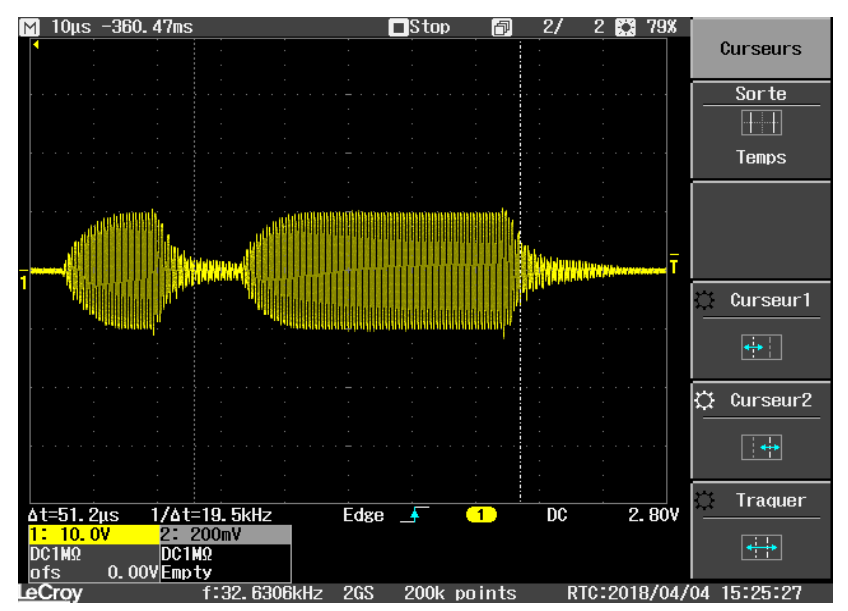

Figure 2. Copy of an oscilloscope screen showing trains of pulses transmitted by a profiler Nortek Group $2 \mathrm{MHz}$ AQP.

The maximum measurable speed $V_{\max }$ depends on $f_{r}$ and on the wavelength $\lambda$ :

$$
\pm V_{\max }=f_{r} \lambda / 4
$$

Zedel and Hay (2010) explain that "measurements can be made at a prescribed range by considering the time elapsed since a sound pulse is transmitted" [24]. It means that $f_{r}$ determines the maximum profiling range $r_{\max }$, at which a target can be detected without ambiguity concerning its position:

$$
r_{\max }=c /\left(2 f_{r}\right)
$$

$c$ is the speed of sound. Relationships (3) and (4) lead to expressing the range-velocity ambiguity relationship [25] as follows:

$$
V_{\max } r_{\max }= \pm c \lambda / 8
$$

To overcome the limits imposed by Equation (5), various techniques have been developed, based on the processing of emitted and received signals.

Thus, conventional profilers are called 'incoherent' or 'narrowband' because the received echoes from two different pulses are not correlated. Echoes are measured continuously, allowing the size of measurement cells in the water column to be determined, considering the value of $c$ and the duration $t_{p}$ of pulses. The lowest uncertainty that can be obtained for the measurements of $\left(V_{1}, V_{2}, V_{3}\right)$ is limited by the standard deviation of the Doppler noise $\sigma_{\delta}$, which is inversely proportional to $t_{p}$. This noise is generated by the random displacement of particles, the multiple echoes and the detection limits of the instrument electronics. To decrease the uncertainty, it is necessary to multiply the number of pulses $n$. The uncertainty on $V_{i}$ 's, $i \in\{1,2,3\}$, can be reduced statistically:

$$
\sigma_{V}=\frac{\sigma_{\delta}}{\sqrt{n}}
$$

Another solution is to increase the value of $t_{p}$, but this leads a reduction in spatial resolution. In order to overcome this ambiguity, 'pulse-to-pulse coherent' or 'pulse coherent' profilers were created. Their measurement principle relies on series of coherent pulses coded in phase. In order to extract the signal from the noise, the autocovariance function of these pulses is calculated [26]:

$$
R(\tau)=C(t) C^{*}(t+\tau)
$$

$C(t)$ is the signal received after the emission of two successive pulses and $C^{*}(t)$ is its complex conjugated expression. To improve the extraction, the autocovariance is assessed from the reception of 
$M$ sequences of two pulses and of the average of $M$ functions $R(\tau)$ [27]. Most often, the average Doppler frequency characterizing the Doppler shift $\delta f_{i}(i \in\{1,2,3\})$ is extracted from the phase $\phi \in[-\pi,+\pi]$ of this average autocovariance function. If $f_{0}$ is the emitted frequency, the measured radial velocity is obtained by the relationship:

$$
V_{i}= \pm \delta f_{i} c /\left(2 f_{0}\right)
$$

According to the Teledyne RD Instrument technical note [28], if $t_{l}$ is the time corresponding to pulses going there and back, we have $2 \pi \delta f_{i}=\phi / t_{l}$. The expression of the velocity becomes:

$$
V_{i}= \pm \phi c /\left(4 \pi f_{0} t_{l}\right)
$$

\section{Perfecting of the Method}

Beyond the complexity of the signal treatment performed by profilers, required in noisy environments that can be found according to particle loads of oceanic profiles, from a metrological point of view it is important to know and to master the possible drift in the frequency or phase of measurements. This drift is conditioned by the quality of the instrument's oscillator and transducer hardware. Therefore, it is necessary to be able to compare the frequency $f_{0}$ emitted by the profiler and the frequencies $f_{0} \pm \delta f_{i}$ resulting from Doppler shifts, to reference frequencies. In fact, the software developed by the manufacturers only gives access to the calculated velocities $V_{i}$ or to referenced velocities $\left(V_{x}, V_{y}, V_{z}\right)$ or $(U, V, W)$.

In a technical note [29], the Teledyne RD Instruments manufacturer proposes fitting a hydrophone on the transducers of its current meters to verify and adjust their electronic gain. Thus, the idea arose to use this kind of hydrophone to simulate echo reception, and to link it to a Rigol DSG3060 frequency generator. Figure 3 shows a RDI hydrophone on two kinds of profilers. Transducer-hydrophone contact is made by silicone gel vacuum grease (Bluesil ${ }^{\mathrm{TM}}$ ) of a relative density 1.0, in order to achieve airtightness. The test bed is located in a laboratory where the temperature is not controlled by air conditioning, but where the temperature is however stable on the short term during measurements. On the long term it can vary of $\pm 1^{\circ} \mathrm{C}$ around $21^{\circ} \mathrm{C}$.

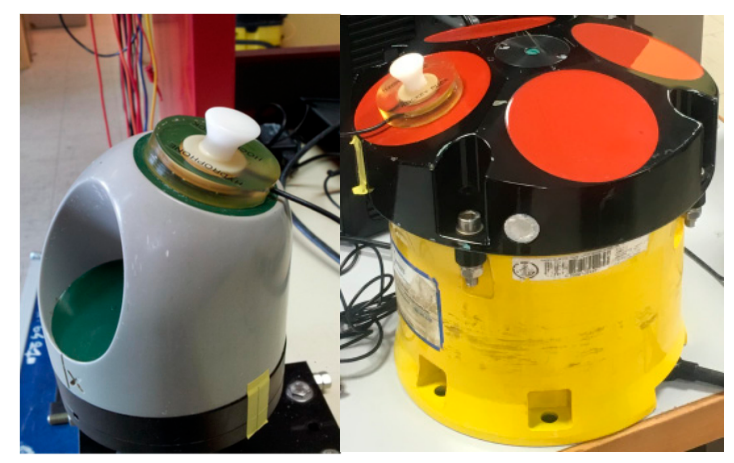

Figure 3. RDI hydrophone on the transducer of a Nortek $600 \mathrm{kHz}$ profiler (on the left) and on the transducer of a Workhorse RDI profiler (on the right).

As current meters and profilers possess different active surfaces and emitting frequencies, according to the type of instrument, it was necessary to use three kinds of plane hydrophone. The first, called RDI, has an active diameter $d$ of $27 \mathrm{~mm}$. Its bandpass is between 400 and $600 \mathrm{kHz}$. For the second, called UB-tr7 from the company Ubertone, it is of $7 \mathrm{~mm}$. Its bandpass is between 1 and $3 \mathrm{MHz}$. Due to the small transducer diameter of some Nortek profilers, a third hydrophone with a diameter of $3 \mathrm{~cm}$ was acquired: RESON TC3027 centered on $1 \mathrm{MHz}$.

Considering the frequency $f_{0}$ of the sinusoidal signal transmitted in the pulses emitted by the profiler, from relationship (8) it is possible to determine a frequency variation range corresponding to its variation range in velocity. It is also possible to determine an increment step $\delta f_{s}$, knowing the 
resolution in velocity $\delta v$ of the instrument. The generator is therefore adjusted to transmit a variable sinusoidal frequency $f_{0} \pm k \delta f_{s}$, the value of $k$ allowing to explore the velocity range.

When the transducer of the profiler is used as a receiver, it sees this signal as an echo. When used as a transmitter, the pulses reflect on the hydrophone but they cannot be detected as their time of arrival is within the blanking distance. The blanking distance corresponds to the time required by the profiler to stop pulse transmission and to switch the transducer to the receiver mode. When the hydrophone does not cover the entire surface of the transducer, one part of the sound wave propagates through air and can be reflected by any objects on its trajectory. The difference between the impedance of the piezo-electrical transducer (which is very high) and that of the air (which is very low), however, results in nearly $99 \%$ of pulse energy being reflected to the interface. The other part, along with pulses transmitted by the profiler's other transducers, can increase the detection noise according to the wavelength of the instrument. All this equipment is remote controlled by a program developed using LabVIEW@ software. It automates testing by decoding the messages from different profiler models, to determine and change their configuration, to extract speed values and to drive the frequency generator.

Using a frequency generator allows the adaptation of the power of the signal heard by the profiler. It can be adjusted to optimize the noise of the measured speeds, taking into account the saturation of the measurement channel. It is also possible to extract the amplitude of the signal (in count) received by each beam, from the messages sent by the instrument. By observing count changes over the long term, it could be possible to monitor changes in current meter sensitivity and then to prevent drift of its amplification hardware.

Relationship (8) includes the speed of sound $c$. When the instrument is used in situ, the user can directly set its value in the software, if measured by a sound velocity sensor, or he can use a relation of calculation based on temperature and pressure measurements made by the profiler (salinity must be determined by another instrument or another method). The issue arose of determining this value in the case of this measurement bed. The value of $c$ was fixed to obtain the broadest possible range of velocities, by staying compatible with the speeds of sound in the ocean. The value $1525 \mathrm{~m} / \mathrm{s}$ was chosen to obtain profiler responses in the range of $\pm 6 \mathrm{~m} / \mathrm{s}$ (except for the Nortek versions called DeepWater where $f_{r}$ is different and leads phase wrapping). This value is programmed in the instrument and in the LabVIEW program to calculate a reference velocity $V_{\text {ref }}$. It is important that the device under test (DUT) and the LabVIEW program contains the same value of $c$ to see the same speed variations for given frequency variations.

Calibration consists in calculating a speed deviation $\delta v$ from the Doppler effect formula, such that:

$$
\delta v=V_{r e f}-V_{i}=\frac{c}{2}\left(\frac{\delta f_{r e f}-\delta f_{i}}{f_{0}}\right)
$$

with $\delta f_{r e f}=k \delta f_{s} . \delta f_{i}$ is defined by relationship (8), $i$ being the index of the instrument's transducer.

\section{Speed Measurement and Calibration Uncertainties}

Current meter oscillators can drift over time and with temperature variations. In order to use relationships (8) and (10), an initial problem arises with the determination of $f_{0}$. The manufacturer Nortek indicates in its documentation that a given number of parameters used in the calculations of velocities have fixed values in the instrument. Transducer frequency $f_{0}$ is one of such fixed values. It also indicates that the accuracy of measured velocities should be $1 \%$ of the measured value $\pm 5 \mathrm{~mm} / \mathrm{s}$. This accuracy was probably deliberately underestimated by the manufacturer due to a lack of knowledge of sound velocity in the medium at the time of measurements, the influence of this one being close to $1 \%$ of the measured velocities. It is therefore, a maximum tolerance.

By the calculation of the derivative of $V_{i}$ versus $f_{0}$ from relationship (8), we can see that the relative variations of $f_{0}$ are proportional to the relative variations of $V_{i}$. For example, an $f_{0}$ error of $0.1 \%$ would correspond to an error of $3 \mathrm{~mm} / \mathrm{s}$ for a speed of $3 \mathrm{~m} / \mathrm{s}$. This also corresponds to $400 \mathrm{~Hz}$ for a profiler 
at $400 \mathrm{kHz}$ and $2000 \mathrm{~Hz}$ for a $2 \mathrm{MHz}$ profiler. According to the measurements made with the Shom profilers, the deviations in $f_{0}$ are generally inferior to $0.02 \%$. The influence of the accurate knowledge of its value is therefore negligible for the calculation of $V_{i}$.

The combined uncertainty on speed deviations $\delta v$ has been assessed following the method of the guide for uncertainty measurement (GUM) edited by the BIPM [30]. As the variables in relationship (10) are independent from each other, the combined standard uncertainty $u_{\delta v}$ of the deviations in velocities obtained during the calibration, consist in the quadratic sum's calculation of the partial derivative of each variable multiplied by their estimated uncertainty. The partial derivatives represent in fact, the sensitivity of each variable, and therefore the weight of each variable, to the variations of the output quantity $\delta v$. That gives the following relationship:

$$
u_{\delta v}{ }^{2}=\left(\frac{\partial \delta v}{\delta f_{r e f}}\right)^{2} u_{\delta f r e f}^{2}+\left(\frac{\partial \delta v}{\delta f_{i}}\right)^{2} u_{\delta f i}^{2}+\left(\frac{\partial \delta v}{\delta f_{0}}\right)^{2} u_{f 0}^{2}
$$

where we consider that $c$ is a constant whose value is given for the calculation. This consideration is justified by the fact that for this test, $c$ is a numerical value programmed in the DUT and the LabVIEW program. $u_{\delta f r e f}$ is the uncertainty of the reference frequency, $u_{\delta f i}$ the uncertainty of the Doppler measurement made by the instrument, and $u_{\delta f 0}$ the uncertainty of the emitted frequency. Relationship (11) gives:

$$
u_{\delta v}^{2}=\left(\frac{c}{2 f_{0}}\right)^{2}\left[u_{\delta f r e f}^{2}+u_{\delta f i}^{2}\right]+\left(\frac{\delta v}{f_{0}}\right)^{2} u_{f 0}^{2}
$$

In relationship (12), $u_{\delta f i}$ can be assessed by taking into account the maximum tolerance given by the manufacturer. From relationship (8) we can write:

$$
u_{\delta f i}=\frac{2 u_{v i} f_{0}}{c}
$$

$u_{v i}$ is the uncertainty of the velocity given by the transducer $i$. Expression (12) becomes:

$$
u_{\delta v}^{2}=\left(\frac{c}{2 f_{0}}\right)^{2} u_{\delta f r e f}^{2}+u_{v i}^{2}+\left(\frac{\delta v}{f_{0}}\right)^{2} u_{f 0}^{2}
$$

As explained in Section 5 , it was necessary to measure $f_{0}$ accurately in order to eliminate any errors relating to the principle of the Doppler shift measurement test. Thus, $u_{f 0}$ represents the standard uncertainty of its measurement with a frequency meter. $u_{f 0}$ has been evaluated to be $0.5 \mathrm{~Hz}$. As the numbers given by the manufacturer concerning measurement accuracy are considered as a maximum tolerance, $u_{v i}$ was assessed by dividing them by the square root of 3 , according to the rules of reference [30]. Despite this division, calculations show that this source of uncertainty largely dominates all the others.

\section{Results Obtained}

During the tests, profilers are programmed with an integration time of $1 \mathrm{~s}$, a minimum blanking distance and only one measurement cell to reduce the test duration. As echo reception is not disrupted by the noise inherent to in situ conditions of use, profilers are used in the narrowband mode.

During the initial measurements, the central frequency transmitted by the generator was set to the frequency that the profilers were supposed to emit: $400 \mathrm{kHz}, 600 \mathrm{kHz}, 1 \mathrm{MHz}$, or $2 \mathrm{MHz}$ according to their type. It appeared that the deviation curves we obtained systematically displayed slopes and offsets that varied according to the instrument.

To dispel any doubts concerning the origin of these shifts, measurements were made by progressively varying the frequency $f_{0}$ transmitted by the generator in order to observe its influence on the offsets. As was noticeable, the hydrophone was used as a receiver and connected to a digital 
oscilloscope. A fast Fourier transform (FFT) of the digitized signal revealed frequency shifts of a few hertz to a few tenths of hertz depending on the instrument, and it allowed the accurate measurement of pulse frequencies emitted by the profilers. Figure 4 shows the result of the FFT performed on pulses transmitted by a Nortek Group $2 \mathrm{MHz}$ AQD.

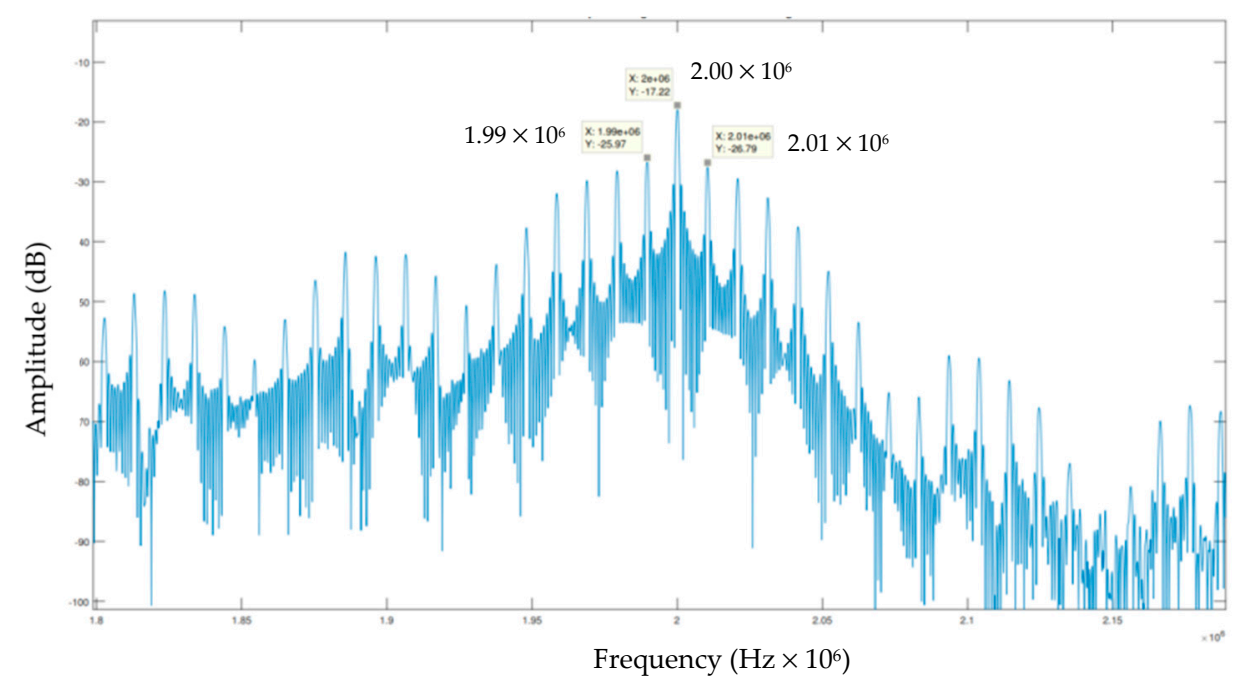

Figure 4. Zoom on the $2 \mathrm{MHz}$ peak obtained by the fast Fourier transform (FFT) of a pulse train emitted by a Nortek Group AQD.

In fact, though the influence of the accurate knowledge of $f_{0}$ on the velocity calculation is negligible, during this test it is necessary to determine it accurately in order to assess the measurement trueness of the Doppler shifts made by the instrument.

Figure 5 shows the result obtained on profiler \# 8691 after adjustment of the frequency transmitted by the generator, on the frequency of the instrument under test. The very low values of measured errors $(<0.002 \mathrm{~m} / \mathrm{s})$ show the accuracy that can be obtained with this test. Concerning Shom's stock of current meters and profilers, most of those that work well, give answers of this type and of this amplitude. Error dashes represent the expanded uncertainty of the calibration obtained with relation (14) and expressed with a coverage factor of 2.

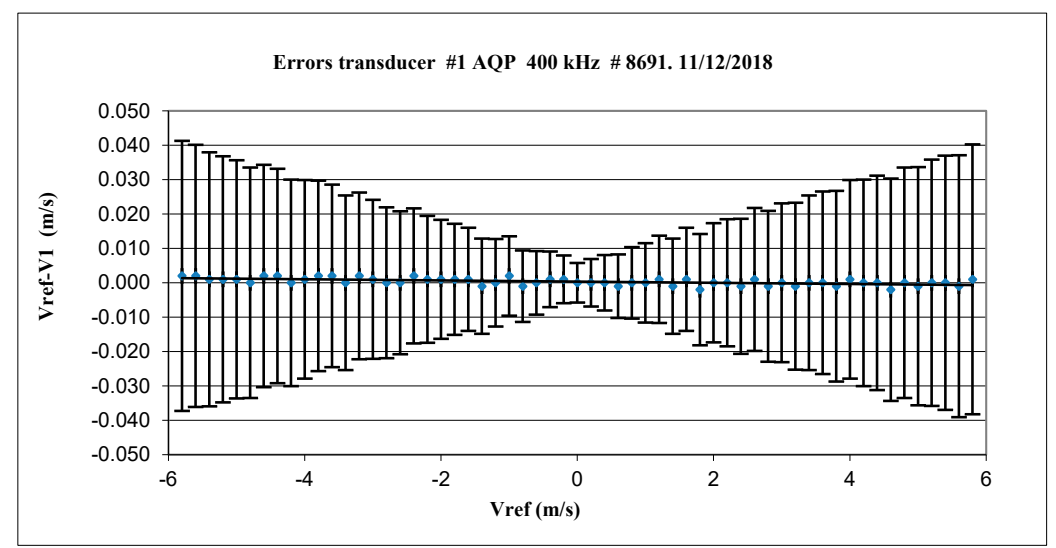

Figure 5. After measurement of its frequency, AQP \# 8691 presents very small shifts. The slope and intercept of the best linear fit (black line) are: $-18 \times 10^{-5} \pm 3 \times 10^{-5}$ and $3 \times 10^{-4} \pm 1 \times 10^{-4} \mathrm{~m} / \mathrm{s}$. Error dashes represent the expanded uncertainty of measurements.

The test procedure was modified to take into account the frequency shifts of the profiler's oscillators. A high-speed digitizer NI USB 5132 was acquired. In order to warrant its stability and 
accuracy in frequency, it was linked to an external Epsilon NTP (Network Time Protocol) clock by TEMEX Telecom, containing a voltage-controlled oscillator (OCXO at $10 \mathrm{MHz})$, synchronized to a GPS signal via an antenna. The resulting average accuracy is $\pm 2 \times 10^{-12}$ at $10 \mathrm{MHz}$ over $24 \mathrm{~h}$. GPS time and frequencies are references directly linking the measurements to the system of units [31]. Figure 6 describes the relationships between the principal elements of the calibration bed.

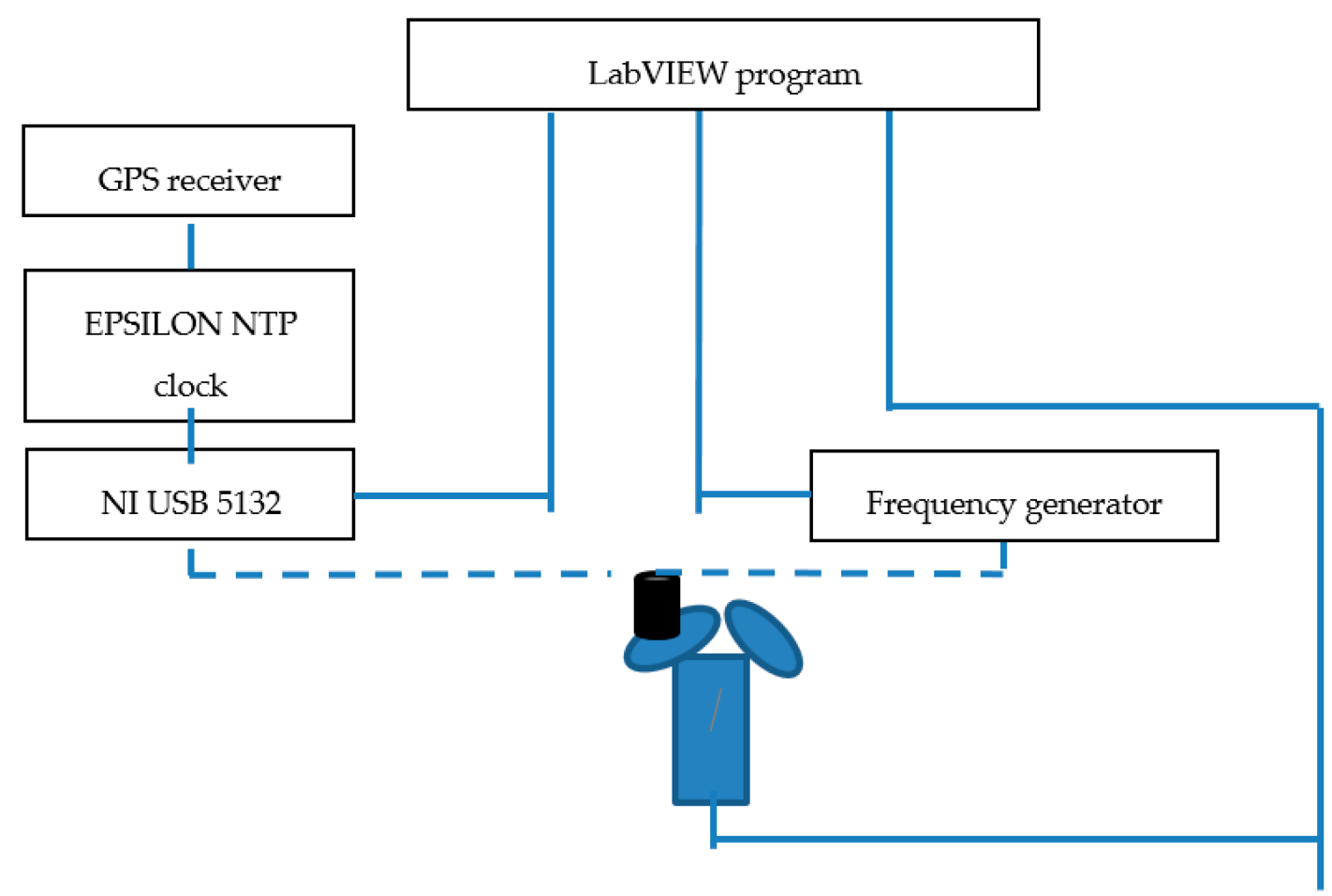

Figure 6. Calibration bed diagram. The hydrophone (in black) is first connected to the NI USB 5132 digitizer whose sampling rate is controlled by an EPSILON NTP clock and a GPS receiver. After obtaining the FFT, it is connected to the frequency generator. Everything is controlled by a Labview (C) program.

About 123 instruments were tested between June 2018 and July 2020 and this test bed allowed faults to be detected on some of them. Table 1 gives statistics of some faults. The line 'AQD' includes current meters $200 \mathrm{~m}, 2000 \mathrm{~m}, 3000 \mathrm{~m}$, and $6000 \mathrm{~m}$. The line 'AQP' includes $400 \mathrm{kHz}, 600 \mathrm{kHz}$, and 1 and $2 \mathrm{MHz}$. Tested AWACS (Acoustic Waves And Current profiler) are $600 \mathrm{kHz}$ and $1 \mathrm{MHz}$. Workhorse are ADCPs $300 \mathrm{kHz}$. The detected defaults are generally minor and the instruments continued to be used except for two of them where at least one transducer was out-of-order (see the text below).

Table 1. Table summarizing the main faults detected, the number of tests per type of instrument and the corresponding percentages. The faults detected are generally minor except for 2 instruments.

\begin{tabular}{ccccccc}
\hline & nb Tested & $\begin{array}{c}\mathbf{0 . 0 1}<\text { Noise } \\
<\mathbf{0 . 0 5} \mathbf{~ m} / \mathbf{s}\end{array}$ & $\begin{array}{c}\text { Non-Linearity } \\
\text { Default }\end{array}$ & $\begin{array}{c}\text { Offset }> \\
\mathbf{0 . 0 1} \mathbf{~ m / s}\end{array}$ & Slope \# 1 & Out-of-Order \\
\hline AQD & 65 & 5 & 2 & 3 & 1 & 0 \\
AQP & 44 & 4 & 0 & 2 & 0 & 2 \\
AWAC & 7 & 2 & 0 & 0 & 0 & 0 \\
Workhorse & 7 & 4 & 0 & 0 & 0 & 0 \\
Total & 123 & 15 & 2 & 5 & 1 & 2 \\
\% & & 12.2 & 1.6 & 4.1 & 0.8 & 1.6 \\
\hline
\end{tabular}

Figures 7 and 8 give examples of noisy transducers found during tests. The origin of this noise is undetermined. Some other transducers present small offsets like in Figure 8, others present small 
non-linearities as in Figure 9. One was found out-of-order and another one with a response completely out of tolerances, as shown in Figure 10. Concerning this AQP, its transducer \#2 was also out of order, though its transducer \#3 was in good working order. This defect could probably have been detected by a towing tank test because of its impact on the calculation of $V_{x}, V_{y}$, and $V_{z}$, though the origin of this fault and the small faults in Figures 7-9 could not have been detected with this accuracy, due to the global test aspect of this method.

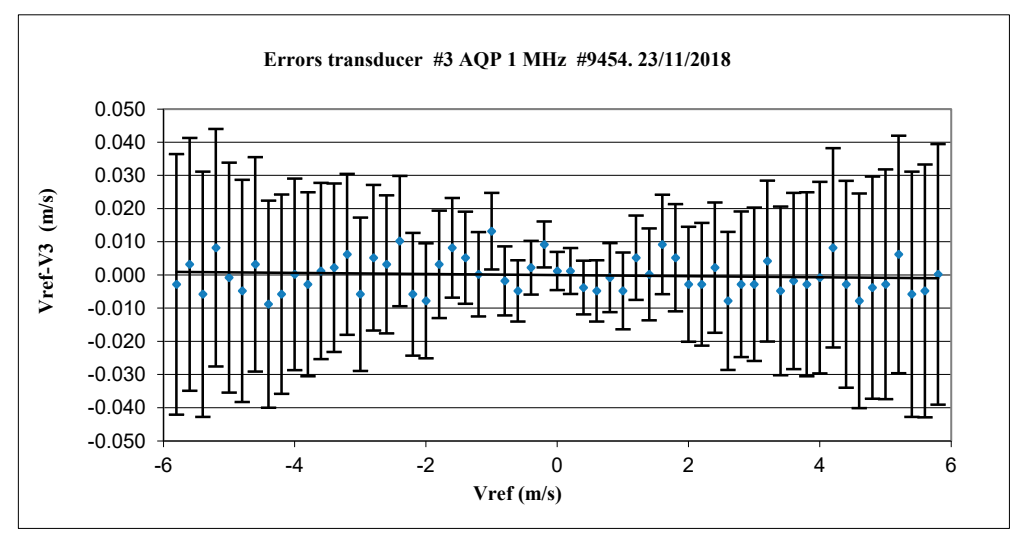

Figure 7. Example of a noisy AQP transducer. The slope and intercept of the best linear fit (black line) are: $-18 \times 10^{-5} \pm 3 \times 10^{-5}$ and $3 \times 10^{-4} \pm 1 \times 10^{-4} \mathrm{~m} / \mathrm{s}$. The noise displays a standard deviation of $0.005 \mathrm{~m} / \mathrm{s}$.

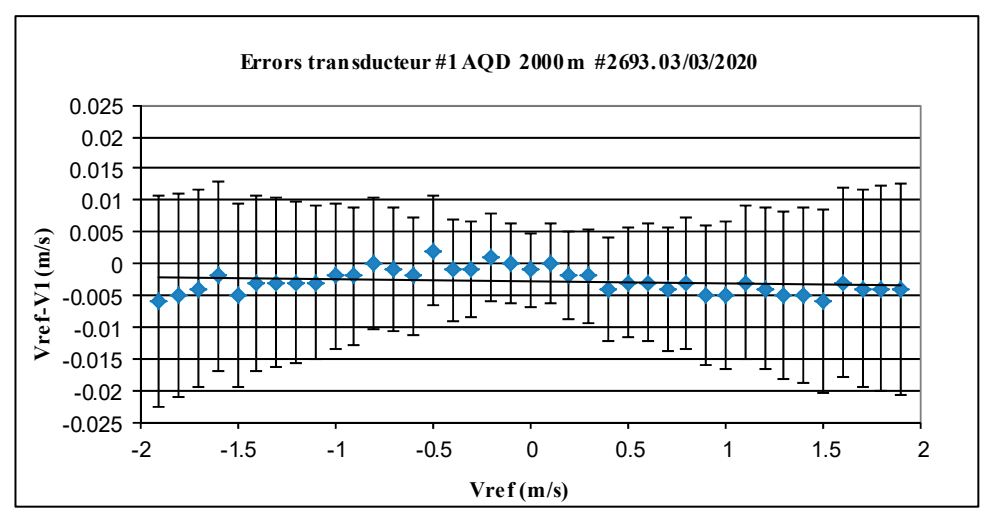

Figure 8. Example of an AQD $2000 \mathrm{~m}$ presenting an average offset of $0.003 \mathrm{~m} / \mathrm{s}$ and noise with a standard deviation of $0.002 \mathrm{~m} / \mathrm{s}$. The slope and intercept of the best linear fit (black line) are: $-3.9 \times 10^{-4} \pm 2.7 \times 10^{-4}$ and $-28 \times 10^{-4} \pm 3 \times 10^{-4} \mathrm{~m} / \mathrm{s}$. The test range is limited to $\pm 2 \mathrm{~m} / \mathrm{s}$ because of phase wrapping led by the different pulse repetition rate of this instrument.

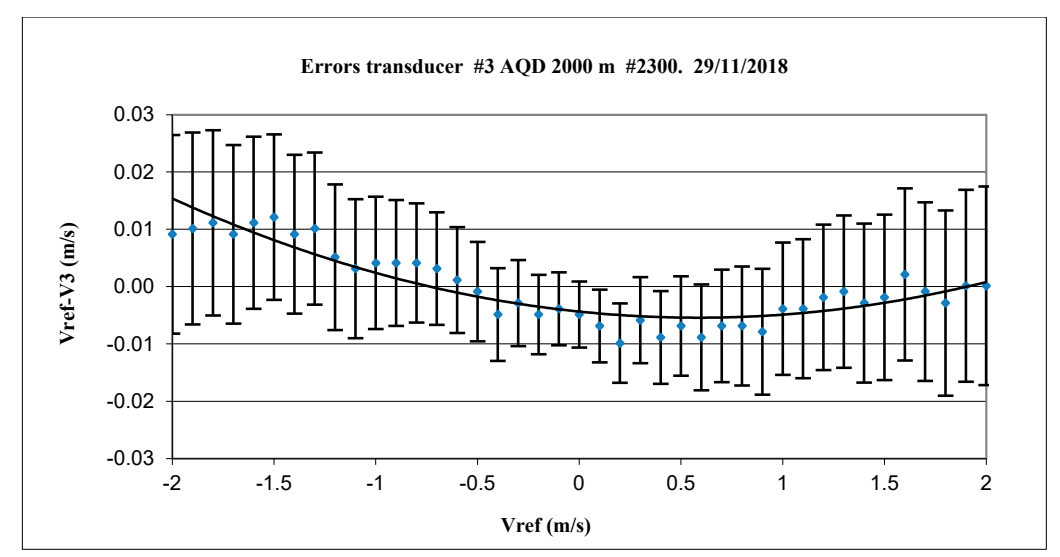

Figure 9. Example of a deep-water AQD transducer with a small non-linearity. 


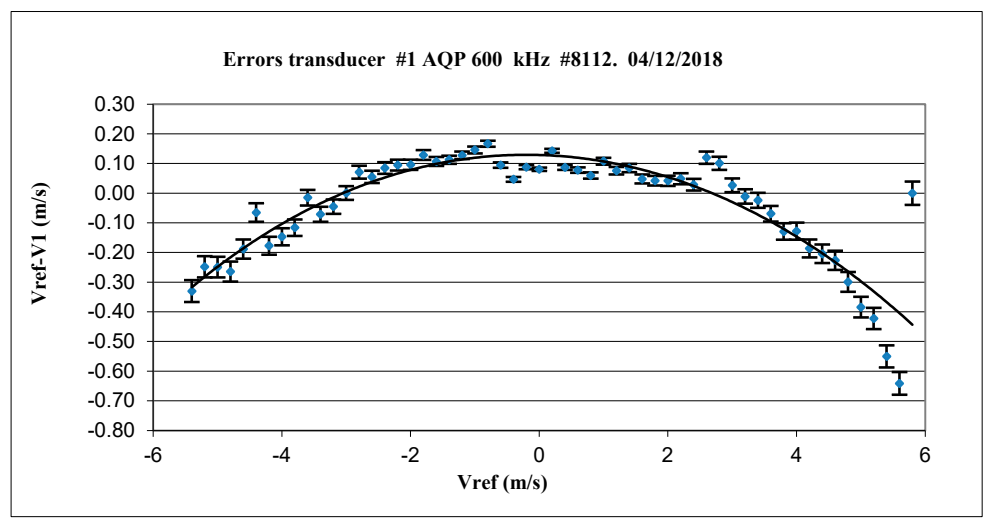

Figure 10. Example of an out-of-order AQP transducer. The response is non-linear and the amplitude of errors is of $0.80 \mathrm{~m} / \mathrm{s}$.

\section{Conclusions}

Current profilers are currently used in large numbers by organizations making oceanographic measurements, but they have been the subject of too few metrological controls, particularly due to their measuring range and because of the complexity of their operating algorithms.

The method perfected, along with the software developed, allows the logging and calibration of most of current meters and profilers manufactured by Nortek AS and RD Instruments. Associated with the compass calibration platform, this test bed allows complete control of the sensors fitted to current meters and current profilers.

It also improved our knowledge of profilers, allowing us in particular to state that the velocity range given in the documentation depends on the speed of sound through the medium. It also allowed us to state that the measurements of the Doppler effect are generally highly reliable. The in situ measurement of uncertainty depends, first and foremost, on the determination of the speed of sound and on particle concentrations along the profiling range of beams. Sound velocity is generally poorly known in places where the profilers are used and over the whole trajectory of pulses. Particle concentrations are also poorly known over the sound range of the instruments, but the high sensitivity of their transducers, the accuracy of the Doppler shift measurements and the complexity of the signal computation made by their software provide sufficiently accurate current speed measurements if their transducers can be shown to function properly using a calibration bed.

Author Contributions: Conceptualization, methodology, writing-original draft preparation, writing-review and editing, M.L.M.; software, validation, S.M. All authors have read and agreed to the published version of the manuscript.

Funding: This research received no external funding.

Acknowledgments: Authors acknowledge André Lusven, Alexandre Léon from SHOM and Sophie Bravo for their technical support.

Conflicts of Interest: The authors declare no conflict of interest. The funders had no role in the design of the study; in the collection, analyses, or interpretation of data; in the writing of the manuscript, or in the decision to publish the results.

\section{Glossary}

$\beta$

$\lambda$

$\Omega$

$\Psi$

$\sigma_{\delta}$

$\sigma_{V}$

$\theta$ transducer slope angle

wavelength

angle of heading

angle of roll

standard deviation of the Doppler noise

standard deviation or uncertainty of velocity $V_{i}$

angle of pitch 


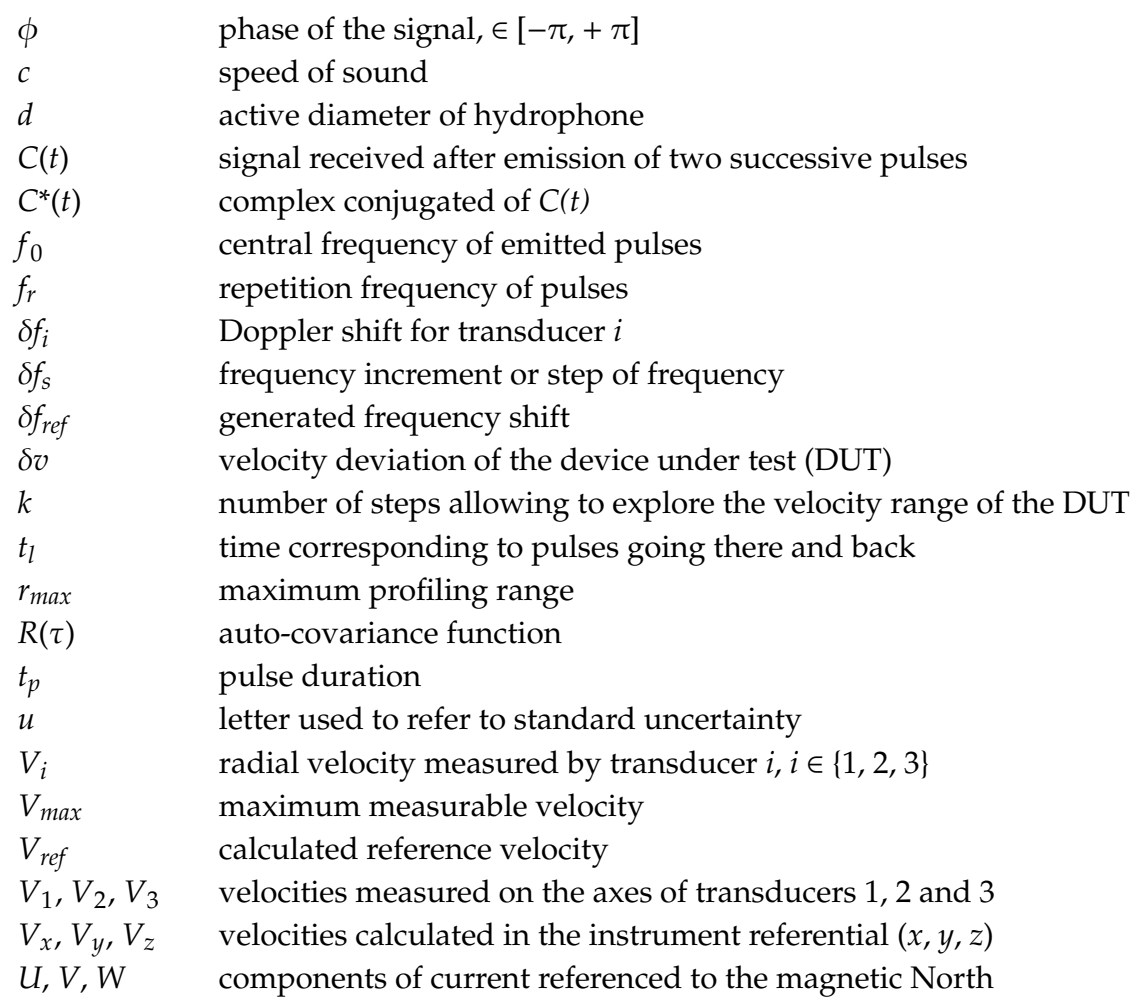

\section{References}

1. Joyce, T.M. On in situ "calibration" of shipboard ADCP. J. Atmos. Ocean. Technol. 1989, 6, 169-172. [CrossRef]

2. Trump, C.L. Calculation of ADCP alignment offsets using single-beam velocity and depth data. J. Atmos. Ocean. Technol. 1997, 14, 1252-1255. [CrossRef]

3. Pollard, R.; Read, J. A method for calibrating shipmounted acoustic Doppler profilers and the limitations of Gyro compasses. J. Atmos. Ocean. Technol. 1989, 6, 859-865. [CrossRef]

4. Trump, C.L.; Marmorino, G.O. Calibrating a gyrocompass using ADCP and DGPS data. J. Atmos. Ocean. Technol. 1997, 14, 211-214. [CrossRef]

5. Pierce, S.D.; Barth, J.A.; Smith, R.L. Improving acoustic Doppler current profiler accuracy with wide-area differential GPS and adaptive smoothing of ship velocity. J. Atmos. Ocean. Technol. 1999, 16, 591-596. [CrossRef]

6. Langlois, G.; Maze, R. Influence of mooring line motion on current measurements. Deep-Sea Res. 1990, 37, 1363-1374. [CrossRef]

7. Munchow, A.; Coughran, C.S.; Hendershott, M.C.; Winant, C.D. Performance and calibration of an acoustic Doppler current profiler towed below the surface. J. Atmos. Ocean. Technol. 1995, 12, 435-444. [CrossRef]

8. Armstrong, B.; Fulford, J.; Thibodeaux, K. Quality assurance testing of acoustic Doppler current profiler transform matrices. In Proceedings of the IEEE/OES 11th Current, Waves and Turbulence Measurement, St. Petersburg, FL, USA, 2-6 March 2015. [CrossRef]

9. Le Coz, J.; Blanquart, B.; Pobanz, K.; Dramais, G.; Pierrefeu, G.; Hauet, A.; Despax, A. Estimating the uncertainty of streamgauging techniques using in situ collaborative interlaboratory experiments. J. Hydraul. Eng. 2016, 142, 7. [CrossRef]

10. Oberg, K.A.; Mueller, D.S. Validation of streamflow measurements made with acoustic Doppler current profilers. J. Hydraul. Eng. 2007, 133, 1421-1432. [CrossRef]

11. Shih, H.H.; Payton, C.; Sprenke, J.; Mero, T. Towing Basin Speed Calibration of Acoustic Doppler Current Profiling Instruments. In Proceedings of the Joint Conference on Water Resource Engineering and Water Resources Planning and Management, Minneapolis, MI, USA, 30 July-2 August 2000. [CrossRef]

12. Le Menn, M.; Pacaud, L. Calibration of currentmeters in direction: Results obtained on a stock of instruments with a new calibration platform. In Proceedings of the 17th International Congress of Metrology, Paris, France, 21-24 September 2015. [CrossRef] 
13. Le Menn, M.; Lusven, A.; Bongiovanni, E.; Le Dû, P.; Rouxel, D.; Lucas, S.; Pacaud, L. Current profilers and currentmeters compass and tilt sensors errors and calibration. Meas. Sci. Technol. 2014, 25, 085801. [CrossRef]

14. Le Menn, M.; Le Goff, M. A method for absolute calibration of compasses. Meas. Sci. Technol. 2007, 18, 1614-1621. [CrossRef]

15. International Organization for Standardization. Assessment of Uncertainty in the Calibration and Use of Flow Measurement Devices_Part 1: Linear Calibration Relationship; International Organization for Standardization: Geneva, Switzerland, 1997; pp. 7066-7071.

16. International Organization for Standardization. Hydrometry-Calibration of Current Meters in Straight Open Tanks; International Organization for Standardization: Geneva, Switzerland, 2007; p. 3455.

17. Camnasio, E.; Orsi, E. Calibration Method of Current Meters. J. Hydraul. Eng. 2011, 137, 386-392. [CrossRef]

18. Boldt, J.A.; Oberg, K.A. Validation of streamflow measurements made with M9 and RiverRay acoustic Doppler current profilers. J. Hydraul. Eng. 2015, 142, 2. [CrossRef]

19. Huang, H. Estimating uncertainty of streamflow measurements with moving-boat acoustic Doppler current profilers. Hydrol. Sci. J. 2018, 63, 3. [CrossRef]

20. JCGM 200:2012. International Vocabulary of Metrology_Basic and General Concepts and Associated Terms, 3rd ed.; BIPM: Saint-Cloud, France, 2012.

21. Cabrera, R.; Huhta, C.; Lohrmann, A. A new acoustic Doppler Current Profiler for Shallow Water. In Proceedings of the MTS/IEEE Conference, Oceans'95, Monterey, CA, USA, 9-12 October 1995; Volume 3, pp. 9-12. [CrossRef]

22. Wertz, J.R. Spacecraft Attitude Determination and Control; Kluwer Academic Press: New York, NY, USA, 1978. [CrossRef]

23. Alderson, S.G.; Cunningham, S.A. Velocity errors in acoustic Doppler current profiler measurements due to platform attitude variations and their effect on volume transport estimates. J. Atmos. Ocean. Technol. 1999, 16, 96-106. [CrossRef]

24. Zedel, L.; Hay, A.E. Resolving velocity ambiguity in multifrequency, pulse-to-pulse coherent Doppler sonar. J. Atmos. Ocean. Technol. 2010, 35, 847-851. [CrossRef]

25. Lhermitte, R.; Serafin, R. Pulse-to-pulse coherent Doppler sonar signal processing techniques. J. Atmos. Ocean. Technol. 1984, 1, 293-308. [CrossRef]

26. Dillon, J.; Zedel, L.; Hay, A.E. Simultaneous Velocity Ambiguity Resolution and Noise Suppression for Multifrequency Coherent Doppler Sonar. J. Atmos. Ocean. Technol. 2012, 29, 450-463. [CrossRef]

27. Lohrmann, A.; Hackett, B.; Roed, L.P. High resolution measurements of turbulence, velocity and stress using a pulse-to-pulse coherent sonar. J. Atmos. Ocean. Technol. 1990, 7, 19-37. [CrossRef]

28. RD Instruments. Field Service Technical Paper 001 (FST-001), Broadband ADCP Advanced Principles of Operation; RD Instruments: San Diego, CA, USA, 1996.

29. RD Instruments. Technical Note FST 004; RD Instruments: San Diego, CA, USA, 1999.

30. JCGM 100:2008. Evaluation of Measurement Data-Guide to the Expression of Uncertainty in Measurement; BIPM: Saint-Cloud, France, 2008.

31. Le Menn, M. Instrumentation and Metrology in Oceanography; ISTE: London, UK; John Wiley \& Sons: Hoboken, NJ, USA, 2012; pp. 38-42. [CrossRef]

Publisher's Note: MDPI stays neutral with regard to jurisdictional claims in published maps and institutional affiliations.

(C) 2020 by the authors. Licensee MDPI, Basel, Switzerland. This article is an open access article distributed under the terms and conditions of the Creative Commons Attribution (CC BY) license (http://creativecommons.org/licenses/by/4.0/). 